\title{
A New Method for Producing Large Carmichael Numbers
}

\author{
By H. Dubner
}

\begin{abstract}
A new method for producing large three-component Carmichael numbers is derived. Only two primes must be found simultaneously instead of three as in the "standard" method. For each set of two primes many third primes can be found. Several Carmichael numbers with more than 3000 digits are shown, with the largest having 3710 digits.
\end{abstract}

1. Introduction. Fermat's "Little Theorem" states:

If $a$ is any integer prime to $N$, and if $N$ is prime, then

$$
a^{N-1} \equiv 1 \quad(\bmod N) \text {. }
$$

Unfortunately, this is not a sufficient condition for a number to be prime since there are composite numbers which satisfy this congruence. Chernick [2] stated the following theorem for these Carmichael numbers:

Fermat's theorem holds for composite integers if and only if $N$ may be expressed as a product of distinct odd primes $P_{1}, P_{2}, \ldots P_{n}$, $(n>2)$, and each $P_{i}-1$ divides $N-1$.

Chernick derived one-parameter expressions for Carmichael numbers which he called "Universal Forms," the most prominent of these being

$$
U_{3}=(6 M+1)(12 M+1)(18 M+1) \text {. }
$$

$U_{3}$ is a Carmichael number when the quantities in parentheses are simultaneously prime. The largest known Carmichael numbers are of this form. In 1980 Wagstaff [6] published a 321-digit Carmichael number. Woods and Huenemann [7] cleverly added a fourth term to Wagstaff's 321-digit number and constructed a four-component Carmichael number with 432 digits.

In August, 1985 the author reported to Wagstaff a 1057-digit number [3], the result of searching for large Carmichael numbers using a specially designed computer for number theory [4]. Since then the author has found, but not reported, a 1265-digit number, a project that took approximately 400 hours on this special computer.

2. The New Method. The difficulty in finding larger Carmichael numbers using Eq. (2) is caused by the necessity of finding three primes simultaneously. The new method is based on finding two primes simultaneously, and then the third prime.

For $N=P Q R, N$ is a Carmichael number if $P, Q, R$ are distinct primes and

$P Q R \equiv 1(\bmod R-1), \quad P Q R \equiv 1(\bmod Q-1), \quad P Q R \equiv 1(\bmod P-1)$.

Received June 17, 1988.

1980 Mathematics Subject Classification (1985 Revision). Primary 11A99. 
It is easy to see that these conditions are equivalent to

$$
P Q \equiv 1(\bmod R-1), \quad P R \equiv 1(\bmod Q-1), \quad Q R \equiv 1(\bmod P-1) .
$$

Next, let $P=6 M+1, Q=12 M+1$, both prime. Then

$$
P Q=(6 M+1)(12 M+1)=72 M^{2}+18 M+1=6 M \cdot 3(4 M+1)+1 .
$$

But

$$
\begin{gathered}
(R-1) X=P Q-1, \\
R=\frac{6 M \cdot 3(4 M+1)}{X}+1 .
\end{gathered}
$$

Also, $P R-1$ must be divisible by $Q-1=12 M$. Now

$$
\begin{aligned}
P R-1= & (6 M+1)\left(\frac{6 M \cdot 3(4 M+1)}{X}+1\right)-1 \\
= & \frac{6 M \cdot 3(4 M+1)(6 M+1)}{X}+6 M=6 M\left(\frac{3(4 M+1)(6 M+1)}{X}+1\right), \\
& \frac{P R-1}{Q-1}=\frac{1}{2}\left(\frac{3(4 M+1)(6 M+1)}{X}+1\right) .
\end{aligned}
$$

Since $6 M+1$ is prime, and considering Eqs. (5) and (6), $X$ must be a divisor of $3(4 M+1)$. The 2 in Eq. (6) will automatically divide since the quantity inside the parentheses is always even. The third condition of Eq. (3) is easily seen to be satisfied.

Thus,

$$
R=6 M k+1,
$$

where $k$ is a divisor of $3(4 M+1)$.

The procedure for searching for large Carmichael numbers is as follows:

1. Find $M$ so that $P=6 M+1$ and $Q=12 M+1$ are prime simultaneously.

2. Find $R=6 M k+1$ prime where $k$ is a divisor of $3(4 M+1)$.

For the method to be productive, the following practical conditions should be met:

1. $M$ should be easily factorable so that $P, Q, R$ can be proven prime using techniques from [1].

2. $4 M+1$ should have many factors so that there are many possibilities for finding $R$ 's that are prime.

Finding functions which fulfill these conditions is like solving a puzzle. The following has been used successfully:

$$
M=\frac{(T C-1)^{A}}{4}
$$

where

$T$ is fixed, odd, and has many factors;

$A$ is fixed and odd;

$C$ is odd and varies until $P$ and $Q$ are prime;

$T C-1$ is less than 24 digits and therefore is easily factored. 
Then,

$$
4 M+1=(T C-1)^{A}+1 .
$$

Since $b^{A}+1$ is always divisible by $b+1$ for $A$ odd, $4 M+1$ will be divisible by $T C$ and therefore has many factors.

Typically, if $T$ is the product of all odd primes through 47 , then $R$ has at least 48,000 possibilities of being prime.

3. Results. It is surprising how easy it is to produce many three-component Carmichael numbers that have two fixed components. For example,

$$
\begin{gathered}
N=P Q R, \quad M=\frac{5^{3} \cdot 7 \cdot 11 \cdot 13-1}{4}=31281, \\
P=6 M+1=187687, \quad Q=12 M+1=375373, \quad R=6 M k+1 ;
\end{gathered}
$$

each of the following values of $k$ gives a prime value of $R$ which makes $N$ a Carmichael number. The list is complete since there are no other such $R$ 's.

\begin{tabular}{rrrr}
\hline$k$ & $R$ & $k$ & $R$ \\
\hline 11 & 2064547 & 231 & 43355467 \\
13 & 2439919 & 385 & 72259111 \\
35 & 6569011 & 825 & 154840951 \\
55 & 10322731 & 975 & 182993851 \\
75 & 14076451 & 3575 & 670977451 \\
77 & 14451823 & 11375 & 2134928251 \\
91 & 17079427 & 25025 & 4696842151 \\
175 & 32845051 & 34125 & 6404784751 \\
\hline
\end{tabular}

In another test, using a 60-digit $M, 501$ prime values of $R$ were found with $k$ limited to a value of one million. Without a limit on $k$, the expected number of prime values of $R$ should exceed $10^{9}$. In other tests, 600-digit Carmichael numbers could be produced at the average rate of one per minute.

The largest Carmichael numbers found appear in Table 1.

\section{TABLE 1}

\section{Parameters for Carmichael Numbers}

$$
\begin{aligned}
N & =P Q R, P, Q, R \text { prime; } \\
P & =6 M+1, Q=12 M+1, R=1+(P Q-1) / X ; \\
M & =(T C-1)^{A} / 4, \\
T & =3 \cdot 5 \cdot 7 \cdot 11 \cdots 43 \cdot 47=307444891294245705 .
\end{aligned}
$$

\begin{tabular}{llllrll}
\hline$A$ & $C$ & $P$ & $Q$ & $X$ & $R$ & $N=P Q R$ \\
\hline 35 & 135449 & 792 digits & 793 digits & 83421 & 1580 digits & 3164 digits \\
& & & & 80073 & 1580 & 3164 \\
41 & 141847 & 929 & 929 & 1172885 & 1852 & 3709 \\
& & & & 803985 & 1852 & 3709 \\
& & & & 325941 & 1852 & 3709 \\
& & & & 217341 & 1852 & 3709 \\
& & & 123165 & 1853 & 3710 \\
\hline
\end{tabular}


It took about twenty hours to find $P$ and $Q$ for the 3710-digit Carmichael number. The expected time was 110 hours for the computer and program used. A new large prime $R$ could be expected about every five hours.

4. The Future. The number of tests (heuristically) required to find $P$ and $Q$ simultaneously prime is proportional to $(\log P)^{2}$. The time to test for probable primality is proportional to $(\log P)^{3}$ using Eq. (1) and standard multiple precision multiplies and divides. Thus, the total time to find $P$ and $Q$ prime is (heuristically)

$$
T=K_{1}(\log P)^{5}=K_{2} D^{5},
$$

where $D$ is the number of digits of $P$. The time to find $R$ can be ignored since it is proportional to only $D^{4}$. The process can be speeded up by using faster computers and more efficient multiplying techniques [5].

It appears possible to extend this new method to further decrease the search time. By choosing $Q=18 M+1$, new equations similar to Eqs. (5) and (6) can be developed but with additional restrictions on $M$ and $X$. It may be possible to eventually develop an algorithm to first find a $P=6 M p+1$, then a $Q=6 M q+1$, then $R=6 M r+1$ so that $P Q R$ is a Carmichael number, but the components have been found sequentially.

Dubner Computer Systems

6 Forest Avenue

Paramus, New Jersey 07675

1. J. BRillhard, D. H. Lehmer \& J. L. SElfridge, "New primality criteria and factorizations of $2^{n} \pm 1$, "Math. Comp., v. 29, 1975, pp. 620-647.

2. J. Chernick, "On Fermat's simple theorem," Bull. Amer. Math. Soc., v. 45, 1939, pp. 269-274.

3. H. Dubner, Letter to S. S. Wagstaff, Jr., dated August 13, 1985.

4. H. DUBNER \& R. DUBNER, "The development of a powerful low-cost computer for number theory applications," J. Recreational Math., v. 18, no. 2, 1985-1986, pp. 81-86.

5. D. E. KNUth, The Art of Computer Programming, Vol. 2. Seminumerical Algorithms, 2nd ed., Addison-Wesley, Reading, Mass., 1981, pp. 278-299.

6. S. S. WAGSTAFF, JR., "Large Carmichael numbers," Math. J. Okayama Univ., v. 22, 1980, pp. 33-41.

7. S. Woods \& J. Huenemann, "Larger Carmichael numbers," Comput. Math. Appl., v. 8, no. 3, 1982, pp. 215-216. 